\title{
Hard Diffraction at the Tevatron and at HERA
}

\author{
Konstantin Goulianos \\ The Rockefeller University \\ 1230 York Avenue, New York, NY 10021, USA
}

ABSTRACT: Experimental results on hard diffraction from HERA and the Tevatron are summarized and compared, and conclusions are drawn about factorization, scaling, the form of the rapidity gap probability in soft and hard diffraction, and the nature of the pomeron.

This paper was adapted from a conference summary talk given at the "Workshop on Diffractive Physics, LAFEX International School on High Energy Physics (LISHEP'98)",10-20 February 1998, Rio de Janeiro, Brazil [hep-ph/9806384!]; it was also presented at the "Fourth Workshop on Quantum Chromodynamics (QCD'98)", 1-6 June 1998, The American Institute of Paris, Paris, France; and at the International Symposium of Multiparticle Dynamics (ISMD'98)", 6-11 September 1998, Delphi, Greece.

\section{Introduction}

Recent results on hard diffraction from HERA and the Tevatron have caused a flurry of theoretical activity. A variety of phenomenological models exist, which have been successful in various degrees in describing the data. However, a QCDbased theoretical description of diffraction is still lacking. This is not surprising, since diffraction invariably involves non-perturbative effects associated with the formation of rapidity gaps. The interplay between soft and hard processes in hard diffraction is of particular theoretical importance due to its potential for elucidating the transition from perturbative to nonperturbative QCD. In this paper, we summarize the main features of the available results on soft and hard diffraction and draw conclusions about the nature of the pomeron, which is presumed to be exchanged in diffractive processes.

\subsection{The signature of hadronic diffraction}

The generic signature of diffraction, which applies to all soft and hard diffractive processes, is the presence of one (or more) rapidity gap(s) in an event, whose probability of formation is not exponentially damped. Generally, the exchange of a gluon, a quark or a color-singlet particle state, such as a $\rho$-meson, between two protons at high energies leads to events in which, in addition to whatever hard scattering may have occured, the entire rapidity space is filled with soft (low momentum) particles (underlying event). The soft particle distribution is approximately flat in rapidity. The flat $d N / d Y$ shape is the result of the $x$-scaling $[i]$ of the parton distribution functions of the incoming protons. The total particle multiplicity is given by $N(s)=\int \rho d Y=\rho \ln s$, where $\rho$ is the average particle density in rapidity space and $s$ is in $\mathrm{GeV}^{2}$. Elastic scattering occurs through the Poisson fluctuation of the multiplicity to $N=0$ and therefore is $\sim e^{-\rho \ln s} \sim 1 / s^{\rho}$. Thus, on general field-theoretical grounds, the elastic scattering cross section is expected to fall with increasing energy for any exchange that has quantum numbers other than those of the vacuum, since the acceleration of the field associated with the quantum numbers of the exchange produces radiation that results in a positive particle density $\rho$. The experimental finding that the elastic cross section at high energies not only does not fall but actually increases with energy, led to postulating a new kind of an exchange, the pomeron, defined as a state with the quantum numbers of the vacuum. Since no radiation is emitted by the acceleration of vacuum quantum numbers, pomeron exchange leads to rapidity gaps whose probability is not dampened expo- 
nentially. Therefore, such gaps are an unmistakable signature for diffractive production and can be considered as the generic definition of diffraction. In QCD, the pomeron can be thought of as a color-singlet state of quarks and gluons, whose structure can be probed in hard diffraction processes.

\subsection{Rapidity gap probability distributions}

Rapidity gaps can be formed in all inelastic nondiffractive (ND) events by multiplicity fluctuations. The probability for a gap of width $\Delta Y=$ $Y-Y_{\min }$ within a ND event sample is given by

$$
P_{g a p}^{N D}(\Delta Y)=\rho e^{-\rho \Delta Y}
$$

This probability is normalized so that its integral is unity. A similar expression can be written for the generic gap probability distribution in singlediffraction dissociation (SD),

$$
P_{g a p}^{S D}(\Delta Y)=K \cdot e^{n \Delta Y}
$$

Here, the parameter $n$ is positive and hence the gap probability grows with increasing $\Delta Y$, in accordance with our generic definition of diffraction. The actual gap probability distribution in a class of diffractive events will be the generic probability modulated by the cross section of the associated pomeron, which generally depends on the width of the gap (see below). The normalization factor $K$ is obtained by setting the integral of the generic gap probability to unity. Such a normalization yields $K=n /\left(e^{n \Delta Y_{\max }}-1\right)$, which for $p p \rightarrow p X$, where $\Delta Y_{\max }=\ln s$, becomes $K=n /\left(s^{n}-1\right) \approx n / s^{n}$. Note that the normalization depends only on $s$ and does not affect the shape of the gap probability.

\subsection{The pomeron flux}

A rapidity gap in $\mathrm{SD}$ is associated with a pomeron, which carries a fraction $\xi$ of the momentum of the proton. The gap width, $\Delta Y$, is related to $\xi$ by

$$
\Delta Y=\ln \frac{1}{\xi}
$$

In terms of $\xi$, Eq. 1.2 becomes

$$
P^{S D}(\xi)=K \cdot \frac{1}{\xi^{1+n}}
$$

Above, we assumed that $n$ is independent of the 4 -momentum transfer, $t$, carried by the pomeron that forms the gap. We now assume, more generally, a linear dependence of $n$ on $t$, which we parametrize as $n(t) \equiv 2\left(\epsilon+\alpha^{\prime} t\right)$, and express $n(t)$ in terms of a new variable, $\alpha(t)$, defined as $\alpha(t) \equiv 1+\epsilon+\alpha^{\prime} t$. Eq. 1.4 takes the form

$$
P^{S D}(\xi, t)=K \cdot \frac{1}{\xi^{2 \alpha(t)-1}} \cdot F^{2}(t)
$$

where we have included the term $F^{2}(t)$, which represents the form factor of the proton, namely the probability that the proton remain intact after receiving a 4 -momentum transfer kick $t$. This formula has exactly the form of the pomeron flux in Regge theory [3], where $\alpha(t)$ is the pomeron trajectory, except for one important difference: since it represents the mapping into $(\xi, t)$-space of the generic gap probability distribution, it must be normalized so that its integral over all available phase space in $\xi$ and $t$ be unity. Such a (re)normalization of the pomeron flux was proposed by this author [i2:

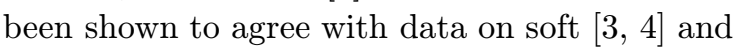

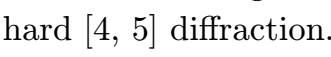

\section{Results}

In this section we examine some experimental results on soft and hard diffraction and compare their main features with those expected from the generic definition of diffraction presented above.

\subsection{Soft Diffraction}

An analysis [ij] of $p p$ and $\bar{p} p$ single diffraction dissociation data has shown that, after subtracting the meson exchange contribution, the cross section can be expressed as a product of the generic gap probability, represented by Eq. 1..5i, times a factor representing the $\mathbb{P}-p$ total cross section, taken (from Regge theory) to be $\sigma^{\mathbb{P} p}=$ $\sigma_{0}^{\mathbb{P} p} \cdot(s \xi)^{\epsilon}$. Note that the gap probability in the $\mathrm{SD}$ event sample is no longer given by Eq. 1.24, since it is modulated by the $\xi^{\epsilon}=e^{-\epsilon \Delta Y}$ dependence of the $\mathbb{P}-p$ total cross section. 


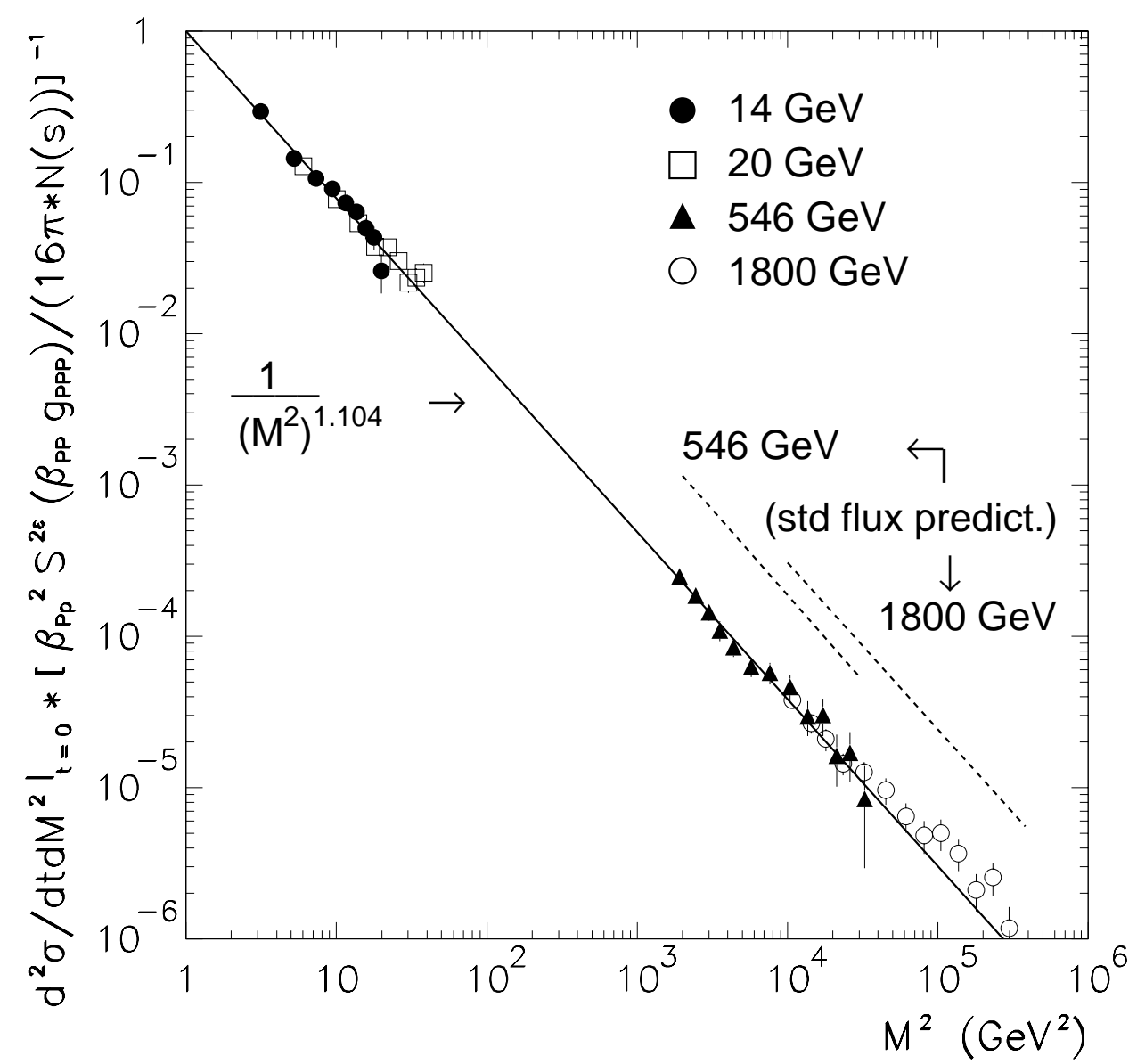

Figure 1: Cross sections $d^{2} \sigma_{s d} / d M^{2} d t$ for $p+p(\bar{p}) \rightarrow p(\bar{p})+X$ at $t=0$ and $\sqrt{s}=14,20,546$ and $1800 \mathrm{GeV}$, multiplied by $\left[\beta_{\mathbb{P} p p}^{2} s^{2 \epsilon}\left(\beta_{\mathbb{P} p p} \cdot g_{\mathbb{P} \mathbb{P} \mathbb{P}}\right) /(16 \pi * N(s))\right]^{-1}$, where $N(s)$ is the integral of the pomeron flux, are compared with the renormalized flux prediction of $1 /\left(M^{2}\right)^{1+\epsilon}$ using $\epsilon=0.104$ [1]. The dashed curves show the standard Regge-theory predictions. The $t=0$ data were obtained by extrapolation from their $t=-0.05$ $\mathrm{GeV}^{2}$ values after subtracting the pion exchange contribution [ [ 3 i $]$.

In terms of the diffractive mass squared $M^{2}=$ $\xi s$, the SD cross section at $t=0$ has the form

$\left.\frac{d^{2} \sigma_{s d}}{d M^{2} d t}\right|_{t=0}=\left[\frac{\beta_{\mathbb{P} p}^{2} s^{2 \epsilon}\left(\beta_{\mathbb{P} p} \cdot g_{\mathbb{P} \mathbb{P} \mathbb{P}}\right)}{16 \pi N(s)}\right] \frac{1}{\left(M^{2}\right)^{1+\epsilon}}$

where $N(s)$ is the pomeron flux integral. Figure 1 compares this formula with experimental data [3]. The agreement between theory and data is excellent over six orders of magnitude. For this comparison, we used $\epsilon=0.104$, derived from a global fit to $p^{ \pm}, \pi^{ \pm}$and $K^{ \pm}$elastic and total cross sections and $\rho$-values [1] 13 '

\subsection{Hard Diffraction at HERA}

At HERA, both the $\mathrm{H} 1$ and ZEUS Collaborations used deep inelastic scattering (DIS) to measure the "diffractive structure function" of the proton, $F_{2}^{D(3)}\left(Q^{2}, \beta, \xi\right)$ (integrated over $t$ ), where $\beta$ is the fraction of the momentum of the pomeron taken by the struck quark. Both experiments found the form

$$
F_{2}^{D(3)}\left(Q^{2}, \beta, \xi\right)=\frac{1}{\xi^{1+n}} \cdot A\left(Q^{2}, \beta\right)
$$

in which the variable $\xi$ factorizes out into an expression that has the $\xi$-dependence of the standard Regge theory pomeron flux factor. Therefore, it appeared reasonable to consider the term 
$A\left(Q^{2}, \beta\right)$ as being proportional to the pomeron structure function $F_{2}^{\mathbb{P}}\left(Q^{2}, \beta\right)$. The $A\left(Q^{2}, \beta\right)$ term was found to be rather flat in $\beta$, suggesting that the pomeron has a hard quark structure. For a fixed $\beta, A\left(Q^{2}, \beta\right)$ increases with $Q^{2}$. By interpreting the $Q^{2}$ dependence to be due to scaling violations, the $\mathrm{H} 1$ Collaboration extracted the gluon fraction of the pomeron using the DGLAP evolution equations in a QCD analysis of $F_{2}^{D(3)}\left(Q^{2}\right.$, The ZEUS Collaboration determined the gluon fraction by combining information from diffractive DIS, which is sensitive mainly to the quark component of the pomeron, and diffractive dijet photoproduction, which is sensitive both to the quark and gluon contents. Both experiments agree that the pomeron structure is hard and consists of gluons and quarks in a ratio of approximately 3 to 1 . The extracted gluon fraction does not depend on the normalization of the $F_{2}^{D(3)}\left(Q^{2}, \beta, \xi\right)$ (for H1) or on the normalization of the pomeron flux (for ZEUS).

Addressing now the question of normalization, if the $1 / \xi^{1+n}$ term in Eq. 2.2 represented the generic gap probability, rather than the standard pomeron flux, it should be normalized so that its integral from $\xi_{\min }=Q^{2} / \beta$ s to $\xi_{\max }=$ 1 be unity. Such a normalization yields $K=$ $n\left(Q^{2} / \beta s\right)^{n}$ and therefore $F_{2}^{D(3)}$ can be written as

$$
F_{2}^{D(3)}\left(Q^{2}, \beta, \xi\right) \sim\left[\left(\frac{Q^{2}}{\beta s}\right)^{n} \cdot \frac{1}{\xi^{1+n}}\right] \cdot F_{2}^{\mathbb{P}}\left(Q^{2}, \beta\right)
$$

where the term in brackets represents the generic $\xi$-probability. Since the measured $Q^{2}$ dependence of $F_{2}^{D(3)}$ is represented well [2] by the factor $\left(Q^{2}\right)^{n}$, which belongs to the (pomeron flux) term in the brackets, the pomeron structure,

$F_{2}^{\mathbb{P}}\left(Q^{2}, \beta\right)$, must be largely independent of $Q^{2}$. The asymptotic gluon momentum fractions for a quark-gluon construct for $2 \div 3 \div 4$ quark flavors is [iㄴ] $0.73 \div 0.64 \div 0.57$. The gluon fraction found by ZEUS, $0.3<f_{g}<0.8$, agrees with the fraction expected from the asymptotic rules. The fraction found by $\mathrm{H} 1$ is not relevant if the $Q^{2}$ dependence does not belong to the pomeron structure.

\subsection{Hard Diffraction at the Tevatron}

Both the CDF and DØ Collaborations have re- ported that the jet $E_{T}$ distributions from nondiffractive (ND), single diffractive (SD) and double pomeron exchange (DPE) dijet events have approximately the same shape $[\overline{7}, \overline{1}, \overline{8}, \overline{9}, \overline{9}]$. Assuming a hard pomeron structure, the CDF Collaboration determined the gluon fraction of the pomeron to be $f_{g}=0.7 \pm 0.2$ by comparing the measured rate of diffractive $W$ production, which is sensi$\beta$, 媐).e to the quark content of the pomeron, with the rate for diffractive dijet production, which depends on both the quark and gluon contents. The measured gluon fraction, which is independent of the pomeron flux normalization assumed in the Monte Carlo simulations, agrees with the result obtained by ZEUS.

For a hard pomeron structure with $f_{g}=0.7$ and $f_{q}=0.3$, the measured $W$ and dijet rates are smaller than the rates calculated using the standard pomeron flux by a factor $D=0.18 \pm$ 0.04 . This flux "discrepancy" factor is consistent with the pomeron flux renormalization expecta-

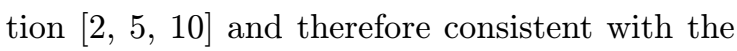
generic $\xi$-distribution probability.

The CDF Collaboration also measured the rate for DPE dijets and compared it with the rates for SD and ND dijets and with calculations using the standard pomeron flux. To obtain the measured DPE/SD ratio, the standard flux in DPE must be multiplied by the factor $D$ for both the proton and antiproton. This result supports the hypothesis that the suppression factor, relative to the standard flux calculations, is associated with the normalization of the $\xi$ probability distribution, rather than with "screening corrections" as proposed by other authors "i6

\section{From HERA to the Tevatron}

The rate for diffractive $W$ production at the Tevatron can be calculated directly from $F_{2}^{D(3)}\left(Q^{2}, \beta, \xi\right)$

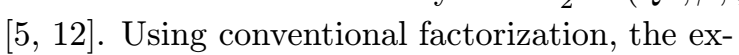
pected SD to ND ratio for $W$ production is $\sim$ $7 \%$. By scaling the $F_{2}^{D(3)}\left(Q^{2}, \beta, \xi\right)$ by the normalization factors of the $1 / \xi^{1+n}$ term at HERA, where $\xi_{\min }=Q^{2} / \beta s$, to that at the Tevatron, where $\xi_{\text {min }}=M_{0}^{2} / \beta s$ with $M_{0}^{2} \approx 1.5 \mathrm{GeV}^{2}$, the prediction becomes $1.24 \%$ 阿, which agrees with the data. This result supports the hypothesis of 
an underlying generic $\xi$ probability distribution given by Eq. 1 - it.

\section{Conclusions}

We have presented a generic definition of diffraction, which is based on the formation of rapidity gaps that are not exponentially damped. The formation of a diffractive rapidity gap is presumed to be associated with the exchange of a pomeron, defined as a color-singlet state with the quantum numbers of the vacuum. The quarkgluon structure of the pomeron can be probed in diffractive processes that incorporate a hard scattering (hard diffraction). Results on hard diffraction from HERA and from the Tevatron indicate that the pomeron structure is composed of gluons and quarks in a ratio of approximately $3 \div 1$. A comparison of hard diffraction rates at HERA with rates at the Tevatron confirms the hypothesis embedded in the generic definition of diffraction that the gap probability must be normalized to unity, i.e. scaled to its integral over all available phase space for gap formation. This scaling of the gap probability violates conventional factorization, but respects unitarity and leads to an unambiguous normalization. Moving beyond phenomenology and providing a QCDbased picture of the pomeron that can explain the experimental results is clearly the next true theoretical challenge.

\section{References}

[1] R. Feynman, 'L Phys. Rev. Lett. $\mathbf{2} 3(1969)$ 1415'.

[2] K. Goulianos, Renormalization of Hadronic Diffraction and the Structure of the Pomeron, P'Pys. Lett. B-358 (1995)) 379; Erratum: Phys.' Lett. B $\mathbf{3 6 3}(1995) 268$

[3] K. Goulianos and J. Montanha, Factorization and scaling in hadronic diffraction [hep-ph/9805496i], Phys. Rev. D ; - - - - - $114017^{\prime}$

[4] K. Goulianos, Pomeron Flux Renormalization: A scaling Law in Diffraction [hep-ph/9806363i].

[5] K. Goulianos, Factorization and scaling in hard diffraction [hep-ph/9708217]; in Proceedings of the 5th International Workshop on Deep Inelastic Scattering and QCD (DIS-97), Chicago, USA, 14-18 April 1997.
[6] E. Gotsman, E.M. Levin and U. Maor, iphys.1 Rev. D 49 (1994) R4321!

[7] M. Albrow, CDF Collaboration, Diffractive dijet production in CDF [FERMILAB-CONF98/134-E].

[8] M. Albrow, CDF Collaboration, "Dijet production by double pomeron exchange in CDF" [FERMILAB-CONF-98/138-E].

[9] B. Hirosky, DØ Collaboration, Dijet production by double pomeron exchange in D $\varnothing$, in Proceedings of "Workshop on Diffractive Physics, LAFEX International School on High Energy Physics (LISHEP-98)," Rio de Janeiro, Brazil, 10-20 February 1998.

[10] K. Goulianos, CDF Collaboration, Diffractive dijet and $\mathrm{W}$ production in CDF [FERMILABCONF-98-117-E].

[11] A. Kaidalov, in Proceedings of "Workshop on Diffractive Physics, LAFEX International School on High Energy Physics (LISHEP-98)," Rio de Janeiro, Brazil, 10-20 February 1998; A. Kaidalov, in Proceedings of "Diffractive production in deep inelastic scattering and hadronic interactions", VII ${ }^{\text {th }}$ Blois Workshop on Elastic and Diffractive Scattering, Château de Blois, France June 20-24 1995, Editions Frontieres (P. Chiappetta, M. Haguenauer and J. Trân Thanh Vân, Eds.), pp. 107-115.

[12] L. Alvero, J. C. Collins J. Whitmore and J. Terron, Phys. Rev. D $\mathbf{5 9}(1999) 074022$.

[13] R.J.M. Covolan, J. Montanha and K. Goulianos, A new determination of the soft pomeron intercept, 'I 